\title{
BMJ Open Assessment of burden of drug-resistant tuberculosis at a tertiary care centre in northern India: a prospective single centre cohort study
}

Richa Misra, ${ }^{1}$ Vasudha Kesarwani, ${ }^{1}$ Alok Nath (1) ${ }^{2}$

To cite: Misra R, Kesarwani V, Nath A. Assessment of burden of drug-resistant tuberculosis at a tertiary care centre in northern India: a prospective single centre cohort study. BMJ Open 2021;11:e044096. doi:10.1136/ bmjopen-2020-044096

- Prepublication history for this paper is available online. To view these files, please visit the journal online (http://dx.doi. org/10.1136/bmjopen-2020044096).

Received 22 August 2020 Revised 26 March 2021 Accepted 30 March 2021

\section{Check for updates}

(C) Author(s) (or their employer(s)) 2021. Re-use permitted under CC BY-NC. No commercial re-use. See rights and permissions. Published by BMJ.

${ }^{1} \mathrm{MD}$, Department of Microbiology, Division Mycobacteriology, SGPGIMS Lucknow, India

${ }^{2} \mathrm{MD}, \mathrm{DM}$, Department of Pulmonary Medicine, SGPGIMS, Lucknow, India

Correspondence to

Dr Alok Nath;

draloksgpgi@gmail.com

\section{ABSTRACT}

Objectives We aim to define the burden of rifampicin monoresistant tuberculosis (TB) at a tertiary care centre in northern India as well as determine the second-line drug susceptibilities (SL-DST) in a subset of patients.

Methods A total of 3045 pulmonary $(n=1883)$ and extrapulmonary $(n=1162)$ samples from likely patients with TB were subjected to microscopy, culture and the Xpert MTB/RIF assay from March 2017 to June 2019. SL-DST testing by line probe assay version 2 for fluoroquinolones (FQs) and second-line injectable drugs were performed on 62 samples.

Results Out of 3045 samples processed in our laboratory during the study period, $36.1 \%(1101 / 3045)$ were positive for Mycobacterium tuberculosis complex (MTBC) and $21.6 \%$ were rifampicin monoresistant (223/1032). The rate of rifampicin resistance in pulmonary samples was $23.5 \%(166 / 706)$ and in extrapulmonary cases, it was $17.4 \%$ (57/326). Out of 62 cases included for second-line testing, 48 were resistant to FQs (77.4\%) while 11 were extensively drug resistant.

Conclusions India urgently needs to arrest an emerging multidrug-resistant TB epidemic with associated resistance to $\mathrm{FQs}$. A robust surveillance system is needed to execute the National Strategic Plan for 2017-2025.

\section{INTRODUCTION}

India has the highest tuberculosis (TB) burden in the world and is home to $26 \%$ of the world's estimated 10.4 million annual TB cases. ${ }^{1}$ The menace of drug resistant-TB (DR-TB), prompted the government to initiate the programmatic management of drug-resistant TB (PMDT) in 2007 which integrates all programme-based strategies for DR-TB diagnosis, management and treatment under the National Tuberculosis Elimination Programme (NTEP) (renamed in December 2019). ${ }^{2}$ India also has a complex as well as unorganised healthcare system which includes the government sector, private sector and informal healthcare providers practicing non-allopathic schools of medicine such as
Strengths and limitations of this study

- Study has a large sample size of 3045 samples

- A total of 1162 extrapulmonary samples such as endobronchial ultrasound with real time-guided transbronchial needle aspiration and biopsies have been included.

- Both Xpert MTB/RIF assay and line probe assay have been performed.

- Mycobacterial Growth Indicator Tube-Drug Susceptibility Testing (MGIT-DST) was not performed.

- DNA sequencing was not done on drug-resistant isolates.

ayurveda and homeopathy. ${ }^{3}$ Though TB was made a notifiable disease in 2012, less than $40 \%$ cases from the private sector were notified to the government in 2017 . $^{4}$

The shorter drug regimen of 9-12 months for patients with multidrug-resistant (MDR)-TB was introduced by WHO, in May 2016 and updated in June 2020. ${ }^{5}$ It was recommended in patients who have not been previously treated with second-line drugs and in whom resistance to fluoroquinolones (FQs) and second-line injectable agents has been excluded. However, drug susceptibility testing in India is technically challenging and requires specialist laboratory facilities and personnel. The TB laboratory network has been expanded over the years to provide better access to quality-assured diagnostic services. Laboratory services are now being provided free of cost to patients attending public health facilities as well as those referred from the private sector. $^{7}$

With this background, we aim to define the burden of rifampicin monoresistant $\mathrm{TB}$ at a tertiary care referral medical centre in northern India as well as determine the second-line drug susceptibilities (SL-DSTs) in a subset of patients. 


\section{METHODS}

\section{Study design and setting}

This prospective observational study between March 2017 and June 2019 was conducted in the Mycobacteriology section of the Department of Microbiology at Sanjay Gandhi Postgraduate Institute of Medical Sciences, a 1200-bed tertiary care referral medical centre in northern India.

\section{Clinical specimens}

Three thousand and forty-five pulmonary and extrapulmonary samples (930 sputum, 752 bronchoalveolar lavage, 146 endobronchial ultrasound with real timeguided transbronchial needle aspiration, 54 bronchial/ tracheal aspirate, 429 lymph node aspirates/fine needle aspiration cytology (FNAC), 367 biopsies, 338 pus and 29 cerebrospinal fluid (CSF) were collected between March 2017 and June 2019 during the clinical routine. All samples were divided into two portions on receipt in the laboratory. One aliquot was used to perform the Xpert MTB/ RIF assay whereas microscopy and culture was performed from the remaining sample. Direct smears were prepared from the specimens using Ziehl-Neelsen staining. All nonsterile clinical samples were processed using the $\mathrm{N}$-acetylL-cysteine-sodium citrate-NaOH method. Samples were decanted following centrifugation, and sediments were resuspended in $3 \mathrm{~mL}$ of phosphate-buffered solution. Processed samples were used to inoculate either Lowenstein-Jensen solid medium or BacT/Alert culture. Line probe assay version 2 (LPAv2) for second-line testing was performed on either direct clinical samples if volume was adequate or on positive culture. Both Xpert MTB/ RIF assay and LPAv2 were performed according to the manufacturer's protocol.

All cases detected positive by the Xpert MTB/RIF assay were grouped into (1) those with smear-positive and culture-positive TB; (2) those with smear-negative, culture-positive TB; (3) those who were both smearnegative and culture-negative for TB but who were nonetheless treated for TB on the basis of clinical, pathological and/or radiological findings (clinical TB).

There was a sub group of samples that were culturepositive but missed by the Xpert MTB/RIF assay. We put up the TB Ag MPT64 Rapid test (SD BIOLINE) on all these positive cultures.

\section{Data collection}

The medical records of patients were retrieved from the hospital information system. A senior resident extracted patient data prospectively from charts.

Classifications and definitions including rifampicinresistant (RR)-TB/MDR-TB/extensively drug resistant (XDR)-TB. ${ }^{8}$

\section{A bacteriologically confirmed TB case}

One from whom a biological specimen was positive by smear microscopy, culture or WHO approved rapid diagnostic test such as Xpert MTB/RIF assay.

\section{Pulmonary TB}

Any bacteriologically confirmed or clinically diagnosed case of TB involving lung parenchyma or tracheobronchial tree.

\section{Extrapulmonary TB}

Any bacteriologically confirmed or clinically diagnosed case of TB involving organs other than the lungs, for example, pleura, lymph nodes, abdomen, genitourinary tract, skin, joints and bones, meninges. Concomitant pulmonary lesions were ruled out in all cases by appropriate investigations and review of case files.

\section{Multidrug-resistant TB}

A patient with TB, whose biological specimen is resistant to both $\mathrm{H}$ and $\mathrm{R}$ with or without resistance to other firstline anti-TB drugs.

\section{Pre-XDR-TB}

It is defined as TB with resistance to isoniazid and rifampicin and either a FQ or a second-line injectable agent but not both.

\section{Extensive drug resistance}

A patient with MDR-TB whose biological specimen is additionally resistant to at least a $\mathrm{FQ}$ and a second-line injectable anti-TB drug.

\section{Patient and public involvement}

Patients were involved in the reporting of our research in this study.

\section{RESULTS}

During the 27-month study period, 1883 pulmonary and 1162 extrapulmonary specimens $(\mathrm{n}=3045)$ were subjected to the GeneXpert MTB/RIF assay in our laboratory along with concomitant smear and culture inoculation on the same sample. All duplicate isolates were excluded. One thousand thirty-two (33.8\%) samples (706 pulmonary, 326 extrapulmonary) were detected for MTB complex. The assay failed to detect 69 samples that were culture-positive. The MPT64 antigen test was positive on all these cultures. There were $806(78.10 \%)$ males and $226(21.89 \%)$ females among the positive specimens. The median age of patients was 32 years and nearly $43 \%$ patients were young adults in the age group of $30-45$ years as shown in figure 1. Lymph node aspirates/ FNAC and tissue biopsy (including colonic biopsy) were

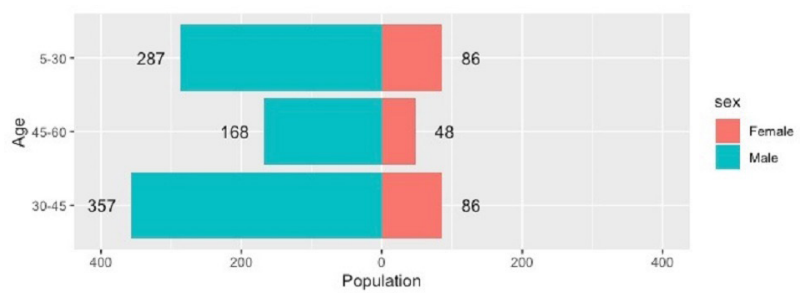

Figure 1 Age distribution of cases positive by Xpert MTB/ RIF assay $(n=1032)$. 


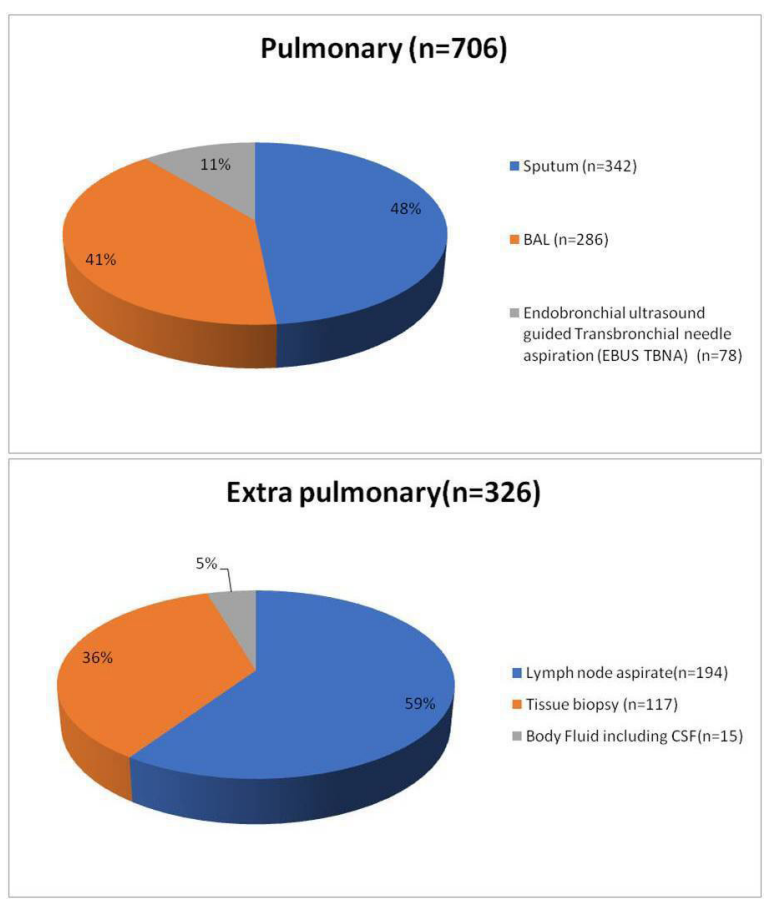

Figure 2 Distribution of samples positive by the Xpert MTB/ RIF assay $(n=1032)$.

the most common samples in extrapulmonary cases that were positive. The sample distribution of positive specimens is shown in figure 2. Out of 1032 samples detected positive by the cartridge based nucleic acid amplification test (CBNAAT) assay, 507 and 517 specimens were smear and culture positive, respectively. The rate of smear and culture positivity in pulmonary and extrapulmonary cases was $54.1 \%, 54.3 \%, 38.3 \%$ and $40.7 \%$, respectively (table 1). The results of conventional and molecular diagnostic testing by Xpert MTB/RIF assay of patients included in the study is shown in figure 3 .

During the study period, we also recovered 34 isolates of non-tuberculous mycobacteria (NTM) from various pus and respiratory specimens. These were Mycobacterium abscessus ( $\mathrm{n}=15), M$. intracellulare (7), M. fortuitum (6), $M$. $\operatorname{gordonae}(\mathrm{n}=3)$ and $M$. simiae $(\mathrm{n}=3)$.

Rifampicin monoresistance was detected in 223 out of 1032 samples $(21.6 \%)$. It was $23.5 \%(\mathrm{n}=166 / 706)$ and $17.4 \%$ (57/326) in pulmonary and extrapulmonary cases, respectively (figure 4). A summary of the performance data is shown in table 2. Five hundred and seventeen samples were positive by culture resulting in an $86.6 \%$ agreement with the Xpert MTB/RIF assay. The assay had

Table 1 Smear and culture results among samples positive by Xpert MTB/RIF assay ( $n=1032)$

\begin{tabular}{lll}
\hline Sample (N=1032) (\%) & Smear-positive & $\begin{array}{l}\text { Culture- } \\
\text { positive }\end{array}$ \\
\hline Pulmonary $(n=706)$ & $382(54.1)$ & $384(54.3)$ \\
Extrapulmonary $(n=326)$ & $125(38.3)$ & $133(40.7)$ \\
Total & $507(49.1)$ & $517(50)$ \\
\hline
\end{tabular}

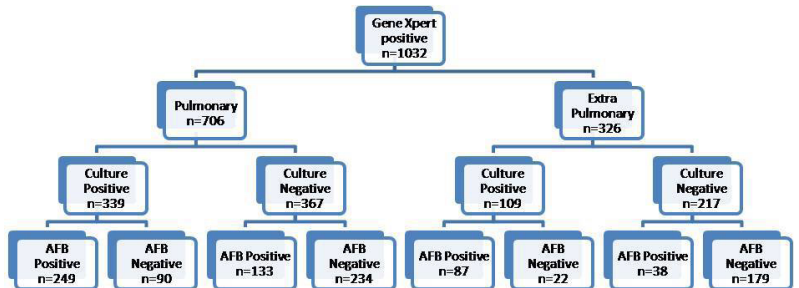

Figure 3 No-Results of conventional and molecular diagnostic testing by Xpert MTB/RIF assay (total number of samples included in the study, $n=3045$ ).

a $100 \%$ agreement for culture-positive, smear-positive specimens and $61.6 \%$ agreement for culture-positive, smear-negative specimens for the detection of M. tuberculosis. Sixty-nine samples that were culture-positive tested negative by the Xpert MTB/RIF assay. We did not have any sample that was positive on both smear and culture but was negative by Xpert MTB/RIF assay. As shown in table 2, we detected 413 more patients than we could have diagnosed by smear and/or culture alone.

Out of 223 rifampicin-resistant cases, we could put up SL-DST testing by LPAv2 for 62 cases $(n=40$, pulmonary and $n=22$, extrapulmonary). As shown in figure 5 , majority of our patients $(77.4 \%)$ were resistant to FQs $(n=48 / 62)$. Only 14 patients were sensitive to both FQ and secondline injectable drug (SLID). Thirty seven cases were resistant to FQs only (Pre-XDR) while 11 were resistant to both classes of drugs (XDR). We did not recover any isolate that was aminoglycoside resistant but FQ sensitive.

\section{DISCUSSION}

MDR-TB is one of the greatest public health challenges worldwide. To the best of our knowledge, ours is the

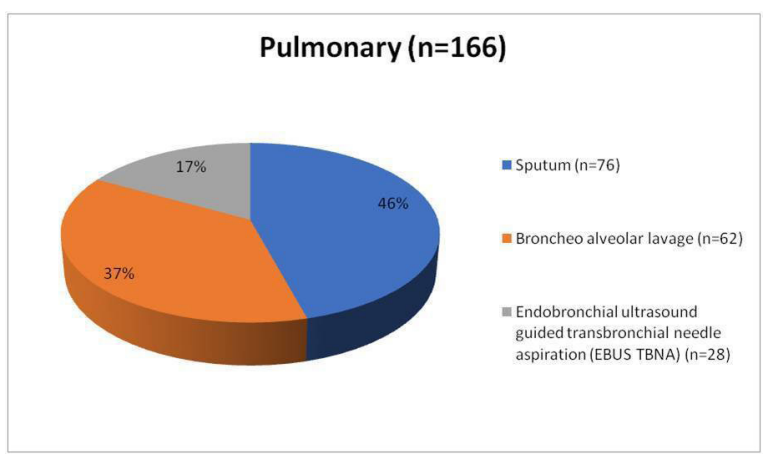

Extra pulmonary $(n=57)$

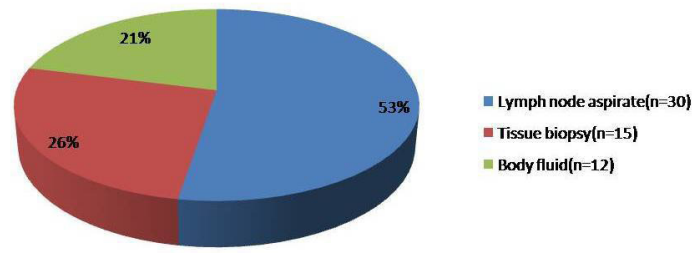

Figure 4 Distribution of samples among rifampicin-resistant cases $(n=223)$. 
Table 2 Comparison of Gene Xpert MTB/RIF positive, MTB culture-positive results with smear results

\begin{tabular}{llllcr}
\hline Smear & $\begin{array}{l}\text { MTB culture + } \\
\text { Gene Xpert+ }\end{array}$ & $\begin{array}{l}\text { MTB culture + } \\
\text { Gene Xpert - }\end{array}$ & $\begin{array}{l}\text { MTB culture- } \\
\text { Gene Xpert+ }\end{array}$ & $\begin{array}{l}\text { MTB culture- } \\
\text { Gene Xpert- }\end{array}$ & $\begin{array}{r}\text { Total } \\
\hline \text { Positive }\end{array}$ 336 \\
Negative & 112 & 69 & 171 & 1944 & 507 \\
Total & 448 & 69 & 413 & 1944 & 2538 \\
\hline
\end{tabular}

first study from India to determine the burden of drugresistant $\mathrm{TB}$ by testing such a large number of pulmonary and extrapulmonary clinical samples. As per Global TB Report 2020, eight countries accounted for two-thirds of the global total: India (26\%), Indonesia $(8.5 \%)$, China $(8.4 \%)$, the Phillippines $(6.0 \%)$, Pakistan $(5.7 \%)$, Nigeria $(4.4 \%)$, Bangladesh $(3.6 \%)$ and South Africa $(3.6 \%) .{ }^{1}$

The results of the national anti-TB drug resistance survey has shown that the incidence of TB is the highest in the age group of 25-34 years in India. ${ }^{9}$ We however documented a slightly higher age group in our study. Our cohort was dominated by males and nearly $43 \%$ patients were young adults in the age group of $30-45$ years. The high frequency of the disease among the younger population may facilitate the transmission of TB in the community due to greater mobility of youth. A gender analysis of the TB epidemic shows that TB affects different genders differently. In 2016, about $40 \%$ of the 2.79 million new cases of TB in India were among women and the male to female ratio for TB stood between 1.07 and 2.25 with women accounting for $40 \%$ of new cases. In our study, it was 3.5. Studies have shown that women may be diagnosed late or not diagnosed at all due to socio-cultural barriers such as high burden of household work, illiteracy, restricted mobility as well as lack of autonomy. There is also a high level of stigma associated with the disease among unmarried females. ${ }^{10}$

WHO's current policies and guidance recommend that the Xpert MTB/RIF assay may be used as an initial diagnostic test in individuals likely of having MDR-TB. About $36 \%$ of the samples included in our study were positive for M. tuberculosis complex and the overall rate of resistance to rifampicin was $21.6 \%$.We assessed the burden of TB in a large cohort of consecutive patients in our hospital thereby eliminating any selection bias in the study population. We also recovered 34 isolates of NTM from various samples, and all these isolates were negative by the Xpert $\mathrm{MTB} / \mathrm{RIF}$ assay.

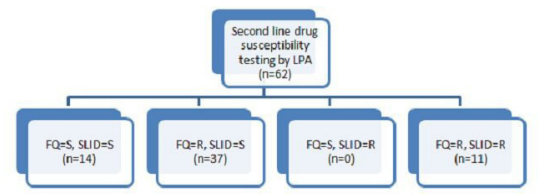

Figure 5 Results for second-line susceptibility testing performed by line probe assay (LPA) version 2. FQ, fluoroquinolones; R, resistant; S, sensitive; SLID, second-line injectable drug.
In a study carried out in Mumbai, India's commercial capital and one of the most densely populated and congested cities, Udwadia et al tested 1539 samples at a tertiary care private hospital and reported MDR-TB in $30.14 \%$ of cases. ${ }^{11}$ In another retrospective study from South India, Shivekar et al performed the MTBDRplus assay on 20245 specimens obtained from presumptive MDR-TB cases during a 6-year study period from 2013 to 2018. Based on the $r p o B$ gene, true resistance, hetero-resistance and inferred resistance to rifampicin was found in $38 \%, 29.3 \%$ and $32.7 \%$ of the 1582 MDR cases, respectively. ${ }^{12}$ Goyal et al published a recent systematic review of 75 epidemiological studies for the prevalence of DRTB in India across two decades, from 1995 to 2015. Comparative analysis revealed a worsening trend in DR-TB between the two study decades, $37.7 \%$ vs $46.1 \%$, respectively. The country-wide prevalence of MDR-TB also increased from the earlier decade at $14.9 \%$ to $27.9 \%$ in decade $2 .{ }^{13}$ However, the report of the first national anti-TB drug resistance survey in India conducted during 2014-2016 concluded that among all patients with TB tested, the MDR-TB rate was $6.19 \%$ with $2.84 \%$ among new and $11.60 \%$ among previously treated patients with TB. The survey has probably under-estimated the true burden of resistance in India since it excluded both smear-negative TB cases as well as extrapulmonary TB and did not include the private sector. ${ }^{9}$

We also attempted to find the overall agreement of the Xpert MTB/RIF assay compared with culture in our study cohort. In this study, the sensitivity of the test was nearly $87 \%$ and it rose to $100 \%$ for smear-positive specimens. The accuracy of the MTB/RIF test to detect the presence of TB in smear-positive cases has been reported to be between $98 \%$ and $100 \% .{ }^{14}$ For smear-negative specimens, Zeka $e t$ al have reported sensitivities of $68.6 \%$ and $47.7 \%$ in pulmonary and extrapulmonary samples, respectively. ${ }^{15}$ Sixty-nine specimens that were culture-positive tested negative by the Xpert MTB/RIF assay in our study resulting in a specificity of $61.6 \%(69 / 112)$. All these samples were smear-negative. We also detected 413 cases ( $40 \%$ of 1032 positives) by the Xpert MTB/RIF assay that were missed by both smear as well as culture. The assay achieved higher diagnostic yield than microscopy and increased TB case finding by a factor of about 2 .

The results of second-line testing in our study revealed $77.4 \%$ resistance to fluroquinolones among $\mathrm{RR}$ isolates which is higher than other studies reported from India. Sethi $e t$ al in a retrospective study from a tertiary care 
centre in northern India have documented an overall rate of $38.6 \% \mathrm{FQ}$ resistance among 863 rifampicin-resistant TB isolates. ${ }^{16}$ In another study from eight healthcare facilities in greater Mumbai between 2005 and 2013, Dalal et $a l$ investigated the trends over time of patterns of drug resistance in a sample of patients with MDR-TB. Between 2005-2007 and 2011-2013, patients with ofloxacin and moxifloxacin resistance significantly increased from $57.6 \%$ to $75.3 \%$ and from $60.0 \%$ to $69.5 \%$ (p<0.05) ${ }^{17} \mathrm{~A}$ meta-analysis by $\mathrm{Ho}$ et al has concluded that globally FQ resistance in MTB is largely confined to MDR strains and knowledge of the global extent of this resistance pattern is currently hampered by the absence of surveillance studies in the majority of regions where TB is endemic. ${ }^{18}$

Updated WHO guidelines, published in June 2020, recommend that for patients with MDR-TB and additional FQ resistance, a regimen composed of bedaquiline, pretomanid and linezolid may be used under operational research conditions (6-9 months). ${ }^{5}$ Chee et al in a study conducted between 2002 and 2016 on 280 patients have demonstrated that only about $30 \%$ of patients with MDR pulmonary TB diagnosed in their study cohort from South-east Asia were eligible for the WHO shorter MDR-TB treatment regimen. ${ }^{19}$ In a similar study from northern India, Singh and Jain have explored the eligibility of the shorter regimen in MDR patients under the programmatic setting. Out of 541 conclusive Line probe assay-second line drugs (LPA-SLD) results, the proportion of strains resistant to only FQs was nearly $50 \%$ while $8.3 \%$ were resistant to both FQs and SLIDs. ${ }^{20}$ Eleven cases in our study were XDR.

The high rates of drug resistance observed in our study may be due to the fact that ours is a tertiary care hospital in the state of Uttar Pradesh which has over 20\% of the total number of notified cases of TB in India. We see patients after the referring hospital has already tried and failed to control infection using a combination of different anti-microbial agents. Since facilities for microbiological studies are usually not available in district hospitals/smaller cities in India, the first contact physician/surgeon/referral facility are compelled to initiate broad-spectrum antibiotics. Indiscriminate antimicrobial therapy without establishing the aetiology of infection selects out the resistant strains. McDowell and Pai in an ethnographic study on the mismanagement of empirical TB treatment in India have demonstrated that all nonspecialist private practitioners began antibiotic treatment, especially quinolones, for persistent cough before prescribing a test. ${ }^{21}$ Their results underscore the fact that inappropriate prescribing practices in India's burgeoning private sector including easy, over-the-counter access to FQs need to be halted as soon as possible.

The alarming rate of drug resistance in our study to rifampicin as well as FQs has important implications for implementation of government strategies to control the TB epidemic in India. First, standardised regimens containing a FQ to treat MDR-TB cases carry a high risk of being suboptimal and resulting in treatment failure.
Second, with such high rates of drug resistance India will have to equip itself with enough mycobacteriology laboratories offering culture and drug susceptibility testing (C-DST) to both first as well as second-line agents. Currently, the focus is to roll out sufficient number of GeneXpert MTB/RIF assay machines to diagnose rifampicin-resistant strains of $M$. tuberculosis. However, this strategy may mask the diagnosis of pre-XDR-TB. A high rate of $\mathrm{FQ}$ resistance has also been noted in newly diagnosed MDR/RR-TB cases, which might be due to transmission of the drug-resistant strains. ${ }^{3}$ It is estimated that in India, by 2032, $85 \%$ of MDR-TB infections would be from primary transmission, compared with only 15\% in 2012. In the Lancet Public Health, Law et al have created a dynamic model of the TB epidemic in India, which they use to estimate the incidence of drug susceptible TB and MDR-TB over the next 20 years. ${ }^{3}$ They have analysed the emergence of drug resistance in all major healthcare sectors in India. Private clinics in India are often used by patients seeking TB treatment and they administer regimens that are not recommended by standard guidelines. This not only results in suboptimal outcome but also potentially generates MDR-TB. They conclude that as MDR-TB transitions from an acquired condition to a primarily transmitted disease, improving the effectiveness of drug-susceptible TB treatment can no longer contain the spread of the epidemic. This epidemiological shift has profound resource implications since the cost of treatment of MDR-TB treatment can exceed that of first-line TB therapy by a factor of 10 or more.

In addition, notification data from low-income and middle-income countries, are prone to underreporting and cannot be interpreted without additional information on case detection rate. The DR-TB diagnostic algorithm as given in the PMDT guidelines recommends second line-line probe assay (SL-LPA) testing for all RR-TB cases diagnosed by the CBNAAT assay. However, it is labour intensive and requires trained manpower. Severe lack of microbiology laboratories providing universal DST and visual interpretation of bands is a huge limitation especially in smear-negative and extrapulmonary cases with inadequate sample volumes as has been our experience even with version 2 of the test.

There were several limitations to this study. One of the methodological limitations of our study was that we could not perform liquid culture DST as well as sequencing and confirm the results of the drug-resistant isolates. Another limitation was that we did not differentiate between new and previously treated TB cases. Most of the patients with DR-TB in our cohort at the time of diagnosis were attached to the PMDT follow-up for further evaluation and management except for some who insisted on institutional management. We could therefore put up SL-DST for only 62 cases. We also did not receive any grant for this study and hence could not put up FL-LPA on the 69 culture-positive isolates that tested negative by the Xpert $\mathrm{MTB} / \mathrm{RIF}$ assay. In addition, a study of risk factors in such 
a high burden setting would have allowed us to offer more useful remedies to policy makers.

\section{CONCLUSION}

In conclusion, we have not come across any prospective study from India on such a large number of pulmonary as well as extrapulmonary samples performed by both conventional and molecular methods. Our study provides comprehensive data on the high burden of drug-resistant TB in India at a 1200-bed tertiary care centre in northern India. The need of the hour is to have enough mycobacteriology laboratories offering both first-line and SL-DST under the NTEP umbrella. The high rates of FQ resistance documented in our study should prompt policy makers to tightly regulate them as reserve drugs, otherwise the ambitious goal of the Government of India to eliminate TB by 2025 seems bleak.

Correction notice This article has been corrected since it was published online. Additional line space in the "RESULTS" section after the word "sample" has been removed.

Acknowledgements The authors thank DK Singh, DC Chamoli, Jyoti Umrao, Santosh and Sadhana for providing technical and logistic support.

Contributors RM conceptualised the manuscript, designed the methods, supervised the study and wrote the manuscript. VK curated the data. AN supervised the study and edited the manuscript. All authors have reviewed the final version of the manuscript.

Funding The authors have not declared a specific grant for this research from any funding agency in the public, commercial or not-for-profit sectors.

Competing interests None declared.

Patient and public involvement Patients and/or the public were involved in the design, or conduct, or reporting or dissemination plans of this research. Refer to the Methods section for further details.

Patient consent for publication Not required.

Ethics approval The study protocol was approved by the Instituitional Ethics Committee of Sanjay Gandhi Post Graduate Institute of Medical Sciences (IEC code 2017-37-IMP-EXP). Written informed consent was obtained from each participant.

Provenance and peer review Not commissioned; externally peer reviewed.

Data availability statement Data are available upon reasonable request. All data relevant to the study are included in the article or uploaded as supplemental information.

Open access This is an open access article distributed in accordance with the Creative Commons Attribution Non Commercial (CC BY-NC 4.0) license, which permits others to distribute, remix, adapt, build upon this work non-commercially, and license their derivative works on different terms, provided the original work is properly cited, appropriate credit is given, any changes made indicated, and the use is non-commercial. See: http://creativecommons.org/licenses/by-nc/4.0/.

ORCID iD

Alok Nath http://orcid.org/0000-0002-7375-5178
REFERENCES

1 World Health Organisation. Global tuberculosis report, 2020. Available: https://www.who.int/tb/publications/global_report

2 Central TB Divison Directorate General of Health Services, Government of India. Programmatic management of drug resistance, 2017, guidelines on programmatic management of drug resistant tuberculosis in India 2017, 2018.

3 Law S, Piatek AS, Vincent C, et al. Emergence of drug resistance in patients with tuberculosis cared for by the Indian health-care system: a dynamic modelling study. Lancet Public Health 2017;2:e47-55.

4 Chatterjee S, Poonawala $\mathrm{H}$, Jain Y. Drugresistanttuberculosis: is India ready for the challenge? BMJ Glob Health 2018;3:e000971.

5 Mirzayev F, Viney K, Linh NN, et al. World Health Organization recommendations on the treatment of drug-resistant tuberculosis, 2020 update. Eur Respir J 2020:2003300

6 Van Deun A, Maug AKJ, Salim MAH, et al. Short, highly effective, and inexpensive standardized treatment of multidrug-resistant tuberculosis. Am J Respir Crit Care Med 2010;182:684-92.

7 Ministry of Health and Family welfare. India TB report 2020: national TB elimination report annual report. central TB divison. Available: www.tbcindia.gov.in

8 WHO. Definitions and reporting framework for tuberculosis 2013 revision (updated Dec 2014 \& Jan 2020). Available: www.who.int

9 Ministry of Health and Family Welfare Gol. Report of the first nationalanti-tuberculois drug resistance survey,2014-16, 2018.

10 Amitapitre. A rapid gender assessment of tuberculosis in India, 2018.

11 Udwadia ZF, Mullerpattan JB, Shah KD, et al. Possible impact of the standardized category IV regimen on multidrug-resistant tuberculosis patients in Mumbai. Lung India 2016;33:253-6.

12 Shivekar SS, Kaliaperumal V, Brammacharry U, et al. Prevalence and factors associated with multidrug-resistant tuberculosis in South India. Sci Rep 2020;10:17552.

13 Goyal V, Kadam V, Narang P, et al. Prevalence of drug-resistant pulmonary tuberculosis in India: systematic review and metaanalysis. BMC Public Health 2017;17:817.

14 Marlowe EM, Novak-Weekley SM, Cumpio J, et al. Evaluation of the Cepheid Xpert MTB/RIF assay for direct detection of Mycobacterium tuberculosis complex in respiratory specimens. J Clin Microbiol 2011;49:1621-3.

15 Zeka AN, Tasbakan S, Cavusoglu C. Evaluation of the GeneXpert MTB/RIF assay for rapid diagnosis of tuberculosis and detection of rifampin resistance in pulmonary and extrapulmonary specimens. $J$ Clin Microbiol 2011;49:4138-41.

16 Sethi S, Agarwal P, Khaneja R, et al. Second-line drug resistance characterization in Mycobacterium tuberculosis by genotype MTBDRsl assay. J Epidemiol Glob Health 2020;10:42-5.

17 Dalal A, Pawaskar A, Das M, et al. Resistance patterns among multidrug-resistant tuberculosis patients in greater metropolitan Mumbai: trends over time. PLoS One 2015;10:e0116798.

18 Ho J, Jelfs P, Sintchenko V. Fluoroquinolone resistance in nonmultidrug-resistant tuberculosis-a surveillance study in New South Wales, Australia, and a review of global resistance rates. Int $J$ Infect Dis 2014;26:149-53.

19 Chee CBE, KhinMar K-W, Sng L-H, et al. The shorter multidrugresistant tuberculosis treatment regimen in Singapore: are patients from South-East Asia eligible? Eur Respir J 2017;50:1700753.

20 Singh PK, Jain A. Limited scope of shorter drug regimen for MDR TB caused by high resistance to fluoroquinolone. Emerg Infect Dis 2019;25:1760-2.

21 McDowell A, Pai M. Treatment as diagnosis and diagnosis as treatment: empirical management of presumptive tuberculosis in India. Int J Tuberc Lung Dis 2016;20:536-43. 